Eye-pointing Classification Scale

\title{
Development and Testing
}

\section{of the Eye-pointing Classification Scale for Children with Cerebral Palsy}

\author{
Michael T. Clarke ${ }^{a^{*}}$, Jenefer Sargent ${ }^{\mathrm{b}}$, Rosemary Cooper ${ }^{\mathrm{a}}$, Gabriella Aberbach $^{\mathrm{a}}$, \\ Laura McLaughlin ${ }^{\mathrm{a}}$, Gurveen Panesar ${ }^{\mathrm{a}}$, Amie Woghiren $^{\mathrm{a}}$, Tom Griffiths ${ }^{\mathrm{c}}$, Katie Price ${ }^{\mathrm{a}}$, \\ Caroline Rose ${ }^{\mathrm{a}}$, John Swettenham ${ }^{\mathrm{a}}$
}

${ }^{a}$ Department of Language and Cognition, University College London, UK

${ }^{b}$ Neurodisability Service, Great Ormond Street Hospital, UK

${ }^{c}$ Communication Aid Service East of England (CASEE, SLT), Addenbrooke's Hospital, UK

\footnotetext{
*Corresponding author: Dr Michael Clarke, Department of Language and Cognition, University College London Chandler House, 2 Wakefield Street, London, WC1N 1PF m.clarke@ucl.ac.uk
} 
Eye-pointing Classification Scale

\begin{abstract}
Purpose

The aim of this study was to develop and test a new classification scale to describe looking behaviours (gaze fixations and gaze shifts) in relation to eye-pointing.

\section{Methods}

The Eye-pointing Classification Scale (EpCS) was developed and tested following established procedures for the construction and evaluation of equivalent scales, and involved 2 phases:

Drawing on research literature, Phase 1 involved initial drafting of the scale through a series of multi-disciplinary group discussions; evaluation of the scale through a survey procedure, and subsequent expert group evaluation. Phase 2, was an examination of scale reliability and relationships between child characteristics and level of EpCS classification.
\end{abstract}

\title{
Results
}

In Phase 1, an initial draft of the scale was developed and then evaluated by 52 participants in 10 countries, leading to its refinement. Further subsequent expert evaluation of content, style and structure indicated that no further refinement was required. In Phase 2, the scale achieved excellent levels of reliability in clinical testing. A significant relationship was identified between level of child motor ability and EpCS classification, and level of child language understanding and EpCS classification.

Keywords: children, eye-pointing, cerebral palsy, classification, communication, vision, joint attention 
Eye-pointing Classification Scale

\section{Acknowledgements}

The authors wish to thank the children, families and clinicians who took part in the research.

This research was generously supported by a grant from Action Medical Research and Great Ormond Street Hospital for Children.

\section{Declaration of interest}

No financial interest or benefit has arisen from the direct applications of this research

\section{Implications for Rehabilitation}

- Non-speaking children with severe bilateral cerebral palsy who have limited upper limb movement may communicate by using controlled looking behaviours to point to objects and people, referred to as eye-pointing.

- However, there is little consensus as to which looking behaviours represent eye-pointing and which do not.

- The Eye-pointing Classification Scale (EpCS) was developed to describe looking behaviours related to eye-pointing in this population of children

- The EpCS provides a new robust tool for clinical management and research with children with cerebral palsy. 


\section{Eye-pointing Classification Scale}

Neuro-typical children begin to show understanding of adult pointing gestures, particularly when preceded by eye contact, and to use pointing actively themselves, around the end of the first year of life [1]. Early pointing behaviors also involve the child shifting gaze between the communication partner and the referenced object [2]. The emergence of finger pointing in young children is of developmental significance for two important reasons. First, it is an indicator of maturing socio-cognitive skills, including triadic joint attention [1]. In its basic form, triadic joint attention concerns co-ordinated attention between two people and an object or action in the environment [3]. It has been proposed also that for joint attention to be fully 'joint', the participants should bring about a co-ordinated orientation to an object or event such that these actions accomplish a shared 'mental state' in the participants [3]. This 'richer' description of joint attention emphasises its intentional, socio-cognitive underpinnings. Finger pointing can be seen as the child's intention to direct attentional focus in an other, and to share a mental state with that person about the pointed to item [4]. A second major reason that pointing is developmentally significant is because of the positive association between children's use of pointing, particularly proto-declarative pointing (pointing to share), and understanding of others' pointing, and both concurrent language ability, and longitudinal language development [5]. Therefore, for clinicians working with children, the emergence of child pointing is a robust developmental marker of significance.

For children with severe bilateral cerebral palsy who have little or no functional speech, and limited upper limb movement preventing manual pointing, controlled looking behaviours, such as gaze fixations and gaze shifts, can be an important component of expressive communication [6]. For example, by shifting gaze between an object and a partner, a child might 


\section{Eye-pointing Classification Scale}

draw a partner's attention to the object in order to make a comment or a request. For clinicians, careful observation of a child's gaze fixations and gaze shifts as a response modality during the presentation of assessment materials can also be the most effective way of determining cognitive and receptive language abilities. Such assessments are particularly important for this group because children with severe bilateral cerebral palsy are known to be at heightened risk of language and cognitive impairments co-occurring with their physical disability [7-9]. Controlled use of gaze fixations and gaze shifts is also a key skill set supporting the use of augmentative and alternative communication (AAC) tools (e.g. looking alternately at graphic symbols on a communication chart and then at a communication partner). Controlled gaze fixations and gaze shifts are also one element of a set of motor and cognitive competences that underpin effective use of gaze-control technologies [10].

The term eye-pointing is often used when describing the controlled use of looking behaviours which can serve the same function as finger pointing in neuro-typical children [6]. Despite the importance of controlled use of gaze fixations and gaze shifts as a communicative strategy by non-speaking children with cerebral palsy, and the relevance of eye-pointing as a potential indicator of developmental progress, evidence indicates that the term eye-pointing has been used inconsistently [6]. Clinical experience also suggests that there is often poor agreement between clinicians concerning which looking behaviors constitute genuine eye-pointing for communication and which do not (e.g. a child may simply happen to look without communicative purpose at one object rather than another, with no intent to point to that object).

The importance of determining the extent to which children with severe bilateral cerebral palsy who have little or no functional speech use looking behaviors for functional communicative purposes is further complicated because these children are known to be at 


\section{Eye-pointing Classification Scale}

increased risk of visual difficulties arising from ocular or cerebral abnormalities. Aspects of cerebral visual impairment (CVI) of particular relevance include eye movement difficulties, reduced visual acuity and impairments in visual attention [11-13]. Furthermore, factors such as pain and motivation may also affect visual responses. For clinicians and families, if weaknesses in functional visual skills are identified then all possible contributory factors will need to be considered.

In order to promote clinical consensus, Sargent and colleagues [14] proposed a description of eye-pointing for communication as: The context-relevant, controlled and intentional use of sustained gaze in order to direct one or more partner's visual attention to any item or object for a deliberate communicative purpose. Other communication modes (facial expression, vocalisation, head movement and body position) may be employed, as available, to support the use of gaze. The intended meaning is established collaboratively between the child and the adult [14, p.479]. Critical elements of this description highlight the active and intentional guiding of others' attention. Eye-pointing therefore not only involves use of controlled gaze fixations and gaze shifts but also the ability to integrate these with social and cognitive abilities as an expression of intent. However, these skills may not develop straightforwardly in all young non-speaking children with bilateral cerebral palsy, and clinicians lack basic practical support to identify evidence of eye-pointing. In the absence of effective practical tools, clinicians may misinterpret children's looking skills relating to eye-pointing, which can lead to inappropriate interventions.

Classification systems to describe movement skills in children with cerebral palsy are well established. The Gross Motor Functional Classification Scale [15], which was the first to be developed, describes gross motor skills, and the Manual Ability Classification System [16], 
Eye-pointing Classification Scale

describes functional hand skills. Classification systems for communication have also been developed and include the Functional Communication Classification System [17], which documents expressive communication skills; the Communication Function Classification System [18], which documents functional abilities in both the receiving and sending of messages; and the Viking Speech Scale [19] which classifies children's speech performance. More recently an Eating and Drinking Classification Scale [20], and a Visual Function Classification System have been published [21]. While the Visual Function Classification System [21] addresses a child's use of vision, it does not distinguish between using vision by seeing (taking in visual information) and using vision by looking (where looking functions as an expressive modality). Such tools have been widely adopted because they facilitate accurate and consistent description of functional abilities in a heterogenous population of children with $\mathrm{CP}$, and offer effective methods of information sharing between clinicians and families, and between clinicians and researchers [22]. However, to date, no tool has been available to characterize critical aspects of looking behavior in relation to eye-pointing for children with severe motor and speech limitations.

The primary aim of the study reported here therefore was to establish and test a valid and reliable descriptive classification scale that would enable clinicians and researchers to describe and categorise looking behaviours related to eye-pointing in the group of children with severe bilateral cerebral palsy who have little or no functional speech and are unable to use limb and hand movements to point effectively. 
Eye-pointing Classification Scale

\section{Method}

The methods for development and validation of the eye-pointing classification scale (EpCS; see table 1) mirrored procedures for the construction and testing of equivalent scales [1521] , and involved two phases: (i) establishing the content validity of the scale; (ii) examining scale reliability and the relationship between child classification and key child characteristics. Ethical approval of this research was granted by the Research Ethics Committee of University College London (UCL 1328/007). The research was funded by Action Medical Research for Children and Great Ormond Street Hospital charity.

Insert table 1 about here

\section{Phase 1: content validity}

The aim of phase 1 was to develop and test the content, style and structure of the scale. Initial drafting of the scale was carried out by a multi-disciplinary development panel. The scale was then evaluated through survey, and was subsequently reviewed by experts at national and international fora.

\section{Participants}

Development Panel: the initial description of the scale was drafted by a panel comprised of three speech and language therapists, one consultant paediatrician, one senior academic in psychology, one clinical scientist, one orthoptist, one optometrist, one former assistant to a child with cerebral palsy, and one parent of a child with cerebral palsy. 


\section{Eye-pointing Classification Scale}

Survey: The survey received anonymous feedback from 52 international participants in 10 countries.

Expert review: The scale was presented for discussion and feedback to 24 multi-disciplinary staff in three specialist centres for the assessment of children with complex needs in the UK, and at two International conferences (Europe and USA).

\section{Procedures}

Development Panel: The panel convened for three 2-hour meetings. Prior to each meeting the latest iteration of the scale was circulated, and the meetings focused on critical review of the content, relevance, and usability of the scale. The group was facilitated by the lead author and proposed amendments agreed by consensus. Meeting notes were taken by a student speech and language therapist in attendance who also contributed to the discussion.

Survey: Feedback was then sought on the scale's content, style, structure and value in relation to clinical relevance through a questionnaire measure distributed to professionals, academics and family members known to the research team, and via fora hosted by Communication Matters (UK Chapter of the International Society of Augmentative and Alternative Communication), and the American Speech and Hearing Association (ASHA). The survey was made available online and consisted of three opening questions seeking information on the participants' relationship to the client group (non-speaking children with cerebral palsy), their geographical location, and how they would define the term 'eye-pointing'. The last question provided an opportunity to determine the participants' degree of suitability for inclusion. No participants were excluded on 


\section{Eye-pointing Classification Scale}

the basis of their responses to this question. Participants were then presented with twenty-one statements concerning the content and usability of the scale and asked to rate their level of agreement with each statement using a seven-point rating scale (strongly disagree to strongly agree; see table S1 supplementary material). A comments box provided with every statement allowed participants to elaborate on their opinions and raise questions with the researchers.

Expert review: Discussion with clinical experts and conference participants centred on the scale's content, style and structure.

\section{Results}

Development Panel: The scale underwent a number of amendments and the final iteration was founded on four key principles. Firstly, the eye-pointing classification scale should reflect the structure of established scales by offering 5 key descriptive categories of functional ability. Secondly, the scale should be used to describe how children typically perform in everyday life through observation of the child in everyday contexts, rather than identifying optimum ability through structured assessment, again reflecting the ethos of similar scales that focus on describing typical levels of functioning [22]. Thirdly, while it is recognised that eye-pointing

represents the combined outcome of the child's visual, social, cognitive and motor abilities [14], from an observer perspective, it is the child's looking behaviours that are the primary expression of eye-pointing. Therefore, the eye-pointing classification scale should focus on describing the child's observable looking behaviours that represent eye-pointing, and on those looking behaviours that fall short of the full expression of eye-pointing but represent a coherent progression of key skills building towards eye-pointing [1,2,23], such as the ability to fix gaze on 


\section{Eye-pointing Classification Scale}

a target and transfer gaze fixations between different targets (see table 1 for full description of the scale). Fourthly, children may eye-point to express a variety of communicative intentions. For example, a child may eye-point to a clock on the wall perhaps to express the word 'clock' or 'time', or to ask the time, or to introduce the concept of time into the current conversation, or to suggest that they wish to finish the current task. Establishing the specific communicative intention of the eye-point can often involve extended and elaborate exchanges between the child and their communication partner. Therefore, the scale should aim to capture the expression of eye-pointing without seeking to document the precise communicative intentions of those looking behaviours.

Survey: The threshold for acceptable levels of agreement between respondents on statements concerning the content, style, structure, and value of the scale was set at $80 \%$ [20]. The twenty statements eliciting feedback from the current study achieved an average level of consensus of $84 \%$. Consensus between respondents fell below $80 \%$ for only four questions: \#2: Five levels cannot adequately describe eye-pointing and its requisite skills (73\% disagreement); \#11: The person using the eye-pointing classification scale to classify a child's looking behaviours must spend time personally interacting with the child in order to provide an accurate level of classification (73\% agreement); \#12: The eye-pointing classification scale should only be used by professionals working with children with disabilities (58\% disagreement); \#13: Where a child's looking behaviours are thought to fall at the borderline between two levels of classification, the level representing the greater degree of limitation should be ascribed (67\% agreement). Examination of written comments relating to these four questions led to an important amendment to the five-point scale which was to allow for its use with children who do 


\section{Eye-pointing Classification Scale}

not appear to fix their gaze consistently on people or objects. Further changes focused on enhancing the written guidance provided with the scale (see: www.ucl.ac.uk/gaze). Expert review: the review centered on use of 5 levels only, and methods for agreeing level of classification, which had achieved lower levels of consensus in the online survey. No further amendments were made to the scale following this phase of evaluation.

\section{Phase 2: Scale Testing}

The aims of phase 2 were to determine (i) the reliability of the scale in use with children with cerebral palsy, and (ii) to examine the relationships between level of classification and other child characteristics including level of language, non-verbal skills and motor disability.

\section{Participants}

47 children ( 21 female, 26 male) were recruited to phase 2 of the study. Criteria for inclusion were that children had a clinical description of bilateral cerebral palsy; chronological age of 3 12 years; little or no functional speech, and were expected to be using vision for learning and communication. In addition, in order to test use of the full range of looking behaviours represented in the scale, children developmentally aged between 1 month and 6 years were recruited. That is, children below and above the developmental age at which finger pointing emerges in neurotypical children [1]. Exclusion criteria were presence of profound hearing impairment, uncontrolled epilepsy, and children who were not expected to be using their vision for learning and communication. Children were recruited from schools in the United Kingdom. School staff were asked to identify children meeting the criteria and to forward information and 


\section{Eye-pointing Classification Scale}

consent procedures to families. Children were visited in school following consent from parents.

Child characteristics are shown in table 2.

Insert table 2 about here

\section{Procedures}

Test-retest reliability was determined by two research assistants (RA1 and RA2) independently classifying each child. In line with other similar tools, the scale was designed to describe children's functional abilities through observation of their performance in ordinary contexts and activities, and the classifications were therefore based on observation of the child in school over the course of a morning or afternoon. A modified test-retest procedure was used. Rather than RA1 and/or RA2 retesting children at a second time point, a third researcher (RA3), who was blind to previous classifications, assessed 41 children at a second visit at least 6 weeks after the first classification. The researchers did not have knowledge of the children prior to assessment. Estimates of language comprehension and non-verbal abilities were derived from direct assessment of children using the Pre-school Language Scale - 4 [24] and the visual receptive scale of the Mullen Scale of Early Learning respectively [25].

\section{Results}

Findings from the analysis of reliability are presented in tables 3 and 4 . Absolute level of agreement between RA1 and RA2 was 81\%. A weighted Cohen's kappa [26] analysis identified an agreement level of $84 \%$ (95\% confidence interval 74\% - 93\%) indicating almost perfect agreement [27]. Intraclass correlation coefficient was 96\% (95\% CI 92\% - 98\%), again demonstrating excellent agreement. Where disagreement was observed it was by no more than 


\section{Eye-pointing Classification Scale}

one level of classification. For example, in the assessment of inter-rater reliability, RA1

classified 10 children at level IV, while RA2 classified 7 children at this level. They disagreed on 3 children as follows: RA1 classified 2 of these children at level IV while RA2 classified them

level III. RA1 also classified 1 child at level IV who was classified at level V by RA2.

Insert tables 3 and 4 about here

The absolute level of agreement between RA2 and RA3 was $80 \%$. The weighted Cohen's kappa test-retest reliability statistic was $79 \%$ (95\% confidence interval $66 \%-93 \%)$, indicating substantial agreement (see table 3) [26]. Again, where there was disagreement it was by no more than one level of classification. Intraclass correlation coefficient was $94 \%$ (95\% confidence interval $88 \%-97 \%)$.

A cumulative odds ordinal logistic regression with proportional odds was run to determine the effect of level of motor ability (GMFCS category IV or V), language age, and nonverbal cognitive age on level of classification on the eye-pointing classification scale. A strong positive correlation was identified between language age and non-verbal cognitive age $r=0.958$, $p<0.0001$, therefore language age only was used in subsequent analyses. While a weak association was observed between GMFCS and language age, an analysis of collinearity indicated that multicollinearity was not a problem for the data set (Variance Inflation Factor $=$ 1.089). The assumption of proportional odds necessary for a robust analysis was also met as assessed by a full likelihood ratio test $\chi 2(6)=3.353, \mathrm{p}=0.7631$ 


\section{Eye-pointing Classification Scale}

A deviance goodness-of-fit test indicated that the model was a good fit to the observed data, $\chi 2(114)=64.772, p=1.000$. The odds of children described as GMFCS V being classified with greater functional limitation on the eye-pointing scale was 4.706, 95\% CI [1.282, 17.274] times that of children classified as GMFCS IV, a statistically significant effect, $\chi 2(1)=5.255, p$ $=.022$. An increase in language age (expressed in years) was associated with an increase in the odds of being classified at a higher level of ability on the eye-pointing classification scale, with an odds ratio of $1.111,95 \%$ CI $[1.056,1.168]$, Wald $\chi 2(1)=16.862, p<.0001$.

\section{Discussion}

The aim of this study was to develop and test a new eye-pointing classification scale suitable for use with non-speaking children with bilateral cerebral palsy (GMFCS IV and V). The scale was established through a 2-stage process that examined the validity and reliability of the new tool. The scale provides a simple description of the potential progression of key looking behaviours that underpin eye-pointing including the ability to establish fixation, disengage and transfer fixation to new items, and to shift fixation between an object of interest and a partner. It also captures the critical elements of eye-pointing itself, which include that it is a controlled and intentional action aimed at drawing others' attention to things in the environment by the child looking alternately at the partner and the item of interest. Thus eye-pointing described in the scale reflects a bid to initiate joint attention. The scale does not document children's motivations for eye-pointing. Children may eye-point to things and people for a host of different reasons and it is the task of the child and their partner together, in that moment, to establish the intended 


\section{Eye-pointing Classification Scale}

function of an eye-point. The scale has been made available free to download at www.ucl.ac.uk/gaze.

Feedback from clinicians highlighted that children's looking behaviours may vary according to the salience of objects in the environment. The current study did not systematically examine changes in children's looking behaviours in the presence of different types of stimuli. Rather, the EpCS was developed and tested to describe how children typically perform in everyday life. This approach reflects the ethos of similar published scales that focus on describing typical levels of functioning [22].

Inter-rater and test-retest reliability was found to be substantial to almost perfect [27]. Where disagreement was observed it was by only one level of classification. Given our modified approach to the assessment of test-retest reliability using a third assessor (RA3) the high level of agreement provides extra confidence that the tool is robust, especially as it is intended to be used by a variety of different professionals/carers. We note that using this modified approach means that it would have been difficult to establish the reasons behind a poor test-retest reliability outcome. Children in this sample were most frequency described as functioning at level III (fixes, disengages and transfers gaze), and II (fixes, disengages, and shifts gaze to face). While $55 \%$ of children in the sample were developmentally aged between 10 months and 6 years 4 months, which is a developmental age at which finger pointing would have been expected to be emerging or fully established in a neuro-typical group, only two children were identified by one of the assessors at level I (uses eye-pointing). The relative rarity of eye-pointing seen in this group may be related to the fact that children were recruited from special schools in the UK. Such children often present with more complex medical, social and learning profiles compared to children with neuro-disabilities in other educational settings. Notably also, a number of children 


\section{Eye-pointing Classification Scale}

were described a functioning at level V (i.e. displays other visual behaviours that do not typically include gaze fixation), despite careful guidance provided to participating schools about inclusion criteria. Reliability testing was carried out by researchers who had experience of working with children with cerebral palsy but who did not know the children involved in the study before visiting them in school. While reliability analysis proved very positive, a limitation of the study is that reliability testing was not also carried out by school staff and parents. The lack of agreement in rating for level $\mathrm{I}$ in this group of children represents a limitation in reliability testing for this level.

A statistically significant relationship was observed between both level of motor ability and language comprehension age, and EpCS classification level. Children with greater gross motor functional limitation were more likely to be classified at a greater level of limitation on the EpCS. Children with higher levels of language comprehension were more likely to be classified at higher levels of ability on the EpCS. One possible explanation for this is that the higher levels of ability in the EpCS reflect joint attention skills which are known to be strongly related to language development in neuro-typical populations [5]. This suggests that similar developmental processes may be involved in language development in this atypical population. At the same time levels III, IV and V do not specifically test joint attention but in this population represent precursors to the expression of joint attention. At these levels the EpCS describes the ability to fix and shift gaze rather than to engage in any socially motivated action. Given the close relation between verbal and non-verbal ability observed in these children, it is likely that level of EpCS classification also reflects a more general level of cognitive functioning. Where difficulties exist in establishing profiles of ability in this population, this simple scale can provide a valuable addition to assessment protocols. 


\section{Eye-pointing Classification Scale}

One potential further use of the scale is to support the targeting of intervention. For example, children functioning at level II (i.e. demonstrating ability to engage with a partner through transfers of gaze from objects to people), may be developmentally ready to progress to expressions of full eye-pointing with suitable intervention. For children at level V, a more appropriate target would to establish ability to fix and hold gaze (Level IV). While the scale has been developed for a sub-group of children with cerebral palsy it could also be used with other populations that also rely on the use of looking behaviours as a primary means of communication. Such groups might include children with Rett syndrome, and adults with acquired motor and communication disorders.

\section{Summary}

The term eye-pointing has been used inconsistently in research and clinical practice. The EpCS was developed to support families and clinicians to systematically describe the ways in which non-speaking children with bilateral cerebral palsy use their looking behaviours functionally for communication. Use of this descriptive scale, along with other robust assessment approaches, has strong potential to empower families and professionals in their clinical decisionmaking [22]. 
Eye-pointing Classification Scale

\section{References}

1. Tomasello M, Carpenter M, Liszkowski U. A new look at infant pointing. Child Dev. 2007 May-Jun;78(3):705-22.

2. Carpenter M, Nagell K, Tomasello M. Social cognition, joint attention, and communicative competence from 9 to 15 months of age. Monogr Soc Res Child Dev. 1998;63(4):i-vi, 1-143.

3. Carpenter M, Liebal K. Joint attention, communication, and knowing together in infancy. Joint attention: New developments in psychology, philosophy of mind, and social neuroscience. 2011:159-181.

4. Liszkowski U, Carpenter M, Henning A, et al. Twelve-month-olds point to share attention and interest. Developmental science. 2004;7(3):297-307.

5. Colonnesi C SG, Koster I, Noom MJ. The relation between pointing and language development: A meta-analysis. Dev Rev. 2010;30(4):352-366.

6. Sargent J, Clarke M, Price K, et al. Use of eye-pointing by children with cerebral palsy: what are we looking at? Int J Lang Commun Disord. 2013 Sep-Oct;48(5):477-85.

7. Venkateswaran S, Shevell MI. Comorbidities and clinical determinants of outcome in children with spastic quadriplegic cerebral palsy. Dev Med Child Neurol. 2008 Mar;50(3):216-22.

8. Stadskleiv K, Jahnsen R, Andersen GL, et al. Neuropsychological profiles of children with cerebral palsy. Dev Neurorehabil. 2018 Feb;21(2):108-120.

9. Rosenbaum P, Paneth N, Leviton A, et al. A report: the definition and classification of cerebral palsy April 2006. Dev Med Child Neurol Suppl. 2007 Feb;109:8-14. 


\section{Eye-pointing Classification Scale}

10. Griffiths T, Addison A. Access to communication technology for children with cerebral palsy. Paediatrics and Child Health. 2017;27(10):470-475.

11. Jan JE, Lyons CJ, Heaven RK, et al. Visual impairment due to a dyskinetic eye movement disorder in children with dyskinetic cerebral palsy. Developmental medicine and child neurology. 2001;43(2):108-112.

12. Costa MF, Ventura DF. Visual impairment in children with spastic cerebral palsy measured by psychophysical and electrophysiological grating acuity tests. Dev Neurorehabil. 2012;15(6):414-24.

13. Sakki HE, Dale NJ, Sargent J, et al. Is there consensus in defining childhood cerebral visual impairment? A systematic review of terminology and definitions. British Journal of Ophthalmology. 2018;102(4):424-432.

14. Sargent J, Clarke M, Price K, et al. Use of eye-pointing by children with cerebral palsy: what are we looking at? [Review]. International Journal of Language \& Communication Disorders. 2013 Sep-Oct;48(5):477-85.

15. Palisano R, Rosenbaum P, Walter S, et al. Development and reliability of a system to classify gross motor function in children with cerebral palsy. Dev Med Child Neurol. 1997 Apr;39(4):214-23.

16. Eliasson AC, Krumlinde-Sundholm L, Rosblad B, et al. The Manual Ability Classification System (MACS) for children with cerebral palsy: scale development and evidence of validity and reliability. Dev Med Child Neurol. 2006 Jul;48(7):549-54.

17. Barty E, Caynes K, Johnston LM. Development and reliability of the Functional Communication Classification System for children with cerebral palsy. Dev Med Child Neurol. 2016 Oct;58(10):1036-41. 


\section{Eye-pointing Classification Scale}

18. Hidecker MJ, Paneth N, Rosenbaum PL, et al. Developing and validating the Communication Function Classification System for individuals with cerebral palsy. Dev Med Child Neurol. 2011 Aug;53(8):704-10.

19. Pennington L, Virella D, Mjøen T, et al. Development of The Viking Speech Scale to classify the speech of children with cerebral palsy. Research in Developmental Disabilities. 2013;34(10):3202-3210.

20. Sellers D, Mandy A, Pennington L, et al. Development and reliability of a system to classify the eating and drinking ability of people with cerebral palsy. Dev Med Child Neurol. 2014 Mar;56(3):245-51.

21. Baranello G, Signorini S, Tinelli F, et al. Visual Function Classification System for children with cerebral palsy: development and validation. Developmental Medicine \& Child Neurology. 2019.

22. Rosenbaum P, Eliasson AC, Hidecker MJ, et al. Classification in childhood disability: focusing on function in the 21st century. J Child Neurol. 2014 Aug;29(8):1036-45.

23. Atkinson J, Braddick O. Visual attention in the first years: typical development and developmental disorders. Dev Med Child Neurol. 2012 Jul;54(7):589-95.

24. Zimmerman IL, Steiner, V.G., Evatt Pond, R. Preschool Language Scale, Fourth Edition (PLS-4 UK). London: Pearson; 2009.

25. Mullen EM. Mullen scales of early learning. AGS Circle Pines, MN; 1995.

26. Kundel HL, Polansky M. Measurement of observer agreement. Radiology. 2003 Aug;228(2):303-8.

27. Landis JR, Koch GG. The measurement of observer agreement for categorical data. Biometrics. 1977 Mar;33(1):159-74. 
Eye-pointing Classification Scale

Table 1. Eye-Pointing Classification Scale

Level I Children at level I demonstrate a consistent ability to fix gaze on

Uses eye-pointing object, disengage gaze from the object, shift and fix gaze on a partner's face, return gaze to fix on the same object and/or fix gaze on partner's face, disengage gaze from their face, shift gaze and fix on and object, then return gaze to fix on same partner's face.

Level II

Shifts gaze to face
Children at level II demonstrate a consistent ability to fix gaze on an object, disengage gaze from the object, shift gaze to a partner's face and/or fixes gaze on partner's face, disengage gaze from their face, and shift gaze to object. Child at level II do not demonstrate triadic gaze shift between objects and people which characterises eyepointing

\section{Level III}

Fixes, disengages

and shifts gaze

Children at level III demonstrate a consistent ability to fixe gaze on objects, disengage gaze from the object, shift gaze away from object and fixes on a new object. Children at Level III do not shift gaze between people and objects.

\begin{tabular}{ll}
\hline $\begin{array}{l}\text { Level IV } \\
\text { Fixes gaze }\end{array}$ & $\begin{array}{l}\text { Children at level IV will demonstrate consistent active ability to fix } \\
\text { and hold gaze on objects or faces but not to obviously shift gaze } \\
\text { between objects or between people. }\end{array}$ \\
\hline Level V & Children at level V will not demonstrate consistent gaze fixation on \\
Other visual & objects or faces (as described in level IV), and/or their visual \\
behaviours & attention to objects or faces cannot be confidently determined.
\end{tabular}


Eye-pointing Classification Scale

Table 2. Characteristics of children participating in the reliability study

\begin{tabular}{|c|c|c|c|c|c|}
\hline & Range & Mean & SD & & \\
\hline Chronological age (yrs: months) & $3: 4-12: 4$ & $8: 4$ & $1: 5$ & & \\
\hline Language age (yrs: months) ${ }^{*}$ & $0: 1-6: 3$ & $1: 8$ & $0: 23$ & & \\
\hline Non-verbal age (yrs: months) ${ }^{* *}$ & $<0: 1-4: 2$ & $1: 1$ & 0.16 & & \\
\hline Number of children per level & Level I & Level II & Level III & Level IV & Level V \\
\hline GMFCS & 0 & 0 & 0 & 19 & 28 \\
\hline MACS & 0 & 1 & 7 & 11 & 28 \\
\hline CFCS & 0 & 3 & 12 & 13 & 19 \\
\hline Viking & 0 & 2 & 8 & 37 & n.a. \\
\hline
\end{tabular}

* Preschool Language Scale, Fourth Edition (PLS-4 UK)

** Mullen scales of early learning

GMFCS, Gross Motor Function Classification System

MACS, Manual Ability Classification System

CFCS, Communication Function Classification System

Viking, Viking Speech Classification System.

$\sim$ The Viking Speech Classification System uses 4 levels only 
Eye-pointing Classification Scale

Table 3. Inter-rater reliability measures for the Eye-pointing Classification Scale

\begin{tabular}{|c|c|c|c|c|c|c|}
\hline & \multicolumn{5}{|c|}{ RA 1 (EPCS level) } & \multirow[b]{2}{*}{ Total } \\
\hline $\begin{array}{c}\text { RA } 2 \\
\text { (EPCS level) }\end{array}$ & $\mathrm{I}$ & II & III & IV & $\mathrm{V}$ & \\
\hline I & 0 & 1 & & & & 1 \\
\hline II & 2 & 8 & 1 & & & 11 \\
\hline III & & 2 & 16 & 2 & & 20 \\
\hline IV & & & & 7 & & 7 \\
\hline V & & & & 1 & 7 & 8 \\
\hline Total & 2 & 11 & 17 & 10 & 7 & 47 \\
\hline
\end{tabular}

The highlighted cells indicate agreement between assessors. 
Eye-pointing Classification Scale

Table 4. Test-retest reliability measures for the Eye-pointing Classification Scale*

\begin{tabular}{|c|c|c|c|c|c|c|}
\hline & \multicolumn{5}{|c|}{ RA 3 (EPCS level) } & \multirow[b]{2}{*}{ Total } \\
\hline $\begin{array}{c}\text { RA } 2 \\
\text { EPCS (level) }\end{array}$ & I & II & III & IV & $\mathrm{V}$ & \\
\hline $\mathrm{I}$ & 0 & 1 & & & & 1 \\
\hline II & & 10 & & & & 10 \\
\hline III & & & 17 & 3 & & 20 \\
\hline IV & & & 3 & 3 & & 6 \\
\hline V & & & & 1 & 3 & 4 \\
\hline Total & 0 & 11 & 20 & 7 & 3 & 41 \\
\hline
\end{tabular}

The highlighted cells indicate agreement between assessors.

*Only those children seen by both RA 2 and RA3 are represented in this table. 
Eye-pointing Classification Scale

Table S1. Survey statements

1. The Eye Pointing Classification Scale [EpCS] is a useful tool; it describes communicative skills in children with cerebral palsy

2. Five levels cannot adequately describe eye pointing and its requisite skills

3. There is good agreement between professionals working with children who have cerebral palsy on what behaviours constitute intentional eye pointing

4. The ability to fix gaze is a fundamental skill required for eye pointing (level V of EpCS)

5. The child's ability to indicate visual recognition is a fundamental skill required for eye pointing (Level IV of EpCS)

6. Disengaging gaze from one object and shifting gaze to another object is a fundamental skill required for eye pointing (Level III of EpCS)

7. Shifting gaze from an object to a face and/or shifting gaze from a face to an object is a fundamental skill required for eye pointing (Level II of EpCS)

8. Eye pointing is most clearly shown when the child fixes their gaze on an object, shifts their gaze to a face, and then returns their gaze to the same object (or vice versa) (Level I of EpCS)

9. The scale provides a good background to the concept of eye pointing

10. Observation of the child over the course of a day is not required for an accurate EpCS rating to be assigned

11. The person using the EpCS to classify a child's looking behaviour must spend time personally interacting with the child in order to provide an accurate level of classification 


\section{Eye-pointing Classification Scale}

12. The EpCS should only be used by professionals working with children with disabilities

13. Where a child's looking behaviours are thought to fall at the borderline between two levels of classification, the level representing the greater degree of limitation should be ascribed.

14. The purpose of the scale is clearly described

15. The EpCS has no clinical value

16. The instructions for the EpCS are clear

17. The distinction between each level of the scale is clear

18. The scale does not take account of key skills used in eye pointing

19. The glossary describing key terms is clear

20. Use of the EpCS will not improve accuracy in describing behaviours related to eye-pointing

21. Use of the EpCS will improve consistency in describing behaviours related to eye-pointing 